\title{
Spectra of Optical-field Ionized Gases by a Femtosecond Ti:Sapphire Laser
}

\author{
Tomas Mocek, Hyun Joon Shin, Yong Ho Cha, Dong Gun Lee, Kyung-Han Hong, and Chang Hee Nam \\ Department of Physics, Korea Advanced Institute of Science and Technology \\ 373-1 Kusong-dong, Yusong-gu, Taejon 305-701, KOREA \\ Hyun Woo Lee \\ Department of Physics, Hanseo University, 353-820 Haemi-myon, Sosan-shi, Chungnam, KOREA
}

(Received July 10, 1998)

\begin{abstract}
We report on the spectroscopic investigation of optical-field ionized plasmas in the soft X-ray spectral region. The experiment was carried out by focusing pulses of the high-power Ti:Sapphire laser with an energy of $\sim 40 \mathrm{~mJ}$ and time duration of $\sim 30$ fs into a gas jet of krypton, xenon, and argon from a pulsed nozzle. Strong soft $\mathrm{X}$-ray emission on lines from ionic stages of $\mathrm{Kr}^{7+}, \mathrm{Kr}^{8+}, \mathrm{Xe}^{7+}, \mathrm{Ar}^{7+}$, and $\mathrm{Ar}^{8+}$ is reported. The experimental result was found to be in good agreement with theoretical prediction.
\end{abstract}

\section{INTRODUCTION}

Recent advances in femtosecond laser technology have opened up new experimental possibilities in high electromagnetic field interaction with matter. The high electric field of the laser pulse can strip electrons from filled atomic shells by the effect of opticalfield ionization (OFI). This ionization mechanism allows control of the stage of ionization by adjusting the intensity of the driving laser and even preparation of a desired electron energy distribution by varying laser polarization. In particular, a linearly polarized laser field generates relatively cold electrons, while circularly polarized radiation produces electrons with high kinetic energy [1].

A systematic study was carried out on the soft Xray emission from $\mathrm{He}, \mathrm{N}_{2}, \mathrm{O}_{2}, \mathrm{CO}_{2}, \mathrm{SF}_{6}$, and $\mathrm{Ar}$ in a gas jet irradiated by 200-fs Ti:Sapphire laser pulses at intensities up to $5 \times 10^{16} \mathrm{~W} / \mathrm{cm}^{2}$ [2]. Recent experiments in which a 250 -fs laser pulse was focused into a low-pressure gas cell ( $\mathrm{He}, \mathrm{O}_{2}, \mathrm{~N}_{2}$, and $\mathrm{CH}_{4}$ at pressures around $1 \mathrm{mbar}$ ) have shown that circular polarization (of the laser) yields a higher electron temperature and thus more efficient excitation than linear polarization does $[3,4]$. Strong X-ray emission from $\mathrm{Ar}$ and $\mathrm{Kr}$ was observed when intense (up to $10^{17} \mathrm{~W} / \mathrm{cm}^{2}$ ) $130-\mathrm{fs}$ pulses were focused into a gas jet under the conditions of clustering [5]. The OFI plasma is also considered as a promising approach for the development of a tabletop soft X-ray laser $(\sim 2-300 \AA)$ [6-9]. Experimentally, gain on the Lyman- $\alpha$ transition of H-like Li at $135 \AA$ was observed $[7,8]$. Another successful approach was reported by a Stanford group [9] on Pd-like Xe at 418 $\AA$ driven by circularly polarized 40 -fs pulses.

In this paper, we present a soft X-ray spectroscopic investigation of optical-field ionized noble gases by a high-power femtosecond Ti:Sapphire laser.

\section{THEORY}

The OFI theory begins with an assessment of the intensity necessary to achieve the desired state of ionization. Although photoionization in very strong fields presents quite a complex theoretical problem, the level of ionization reached during the laser pulse can be estimated by using a simple Coulomb-barrier suppression model (BSI) [10]. This model is based on the superposition of the Coulomb potential of an atom and a quasi-static laser field. As the laser field strength is increased, the height of the potential barrier in the atom is lowered. An electron can be ionized when the lowered atomic potential reaches the ionization potential of the atom (or ion). The corresponding threshold laser intensity can be written as

$$
I_{t h}\left(\mathrm{~W} / \mathrm{cm}^{2}\right)=4 \times 10^{9} \frac{U^{4}(\mathrm{eV})}{Z^{2}}
$$

where $Z$ is the charge of the generated ion and $U$ is the ionization potential of the ion with charge $(Z-1)$. 
For example, in the case of krypton gas, the threshold electric field of $3.4 \times 10^{9} \mathrm{~V} / \mathrm{cm}$ is required for generation of a Ni-like charge state $\left(\mathrm{Kr}^{8+}\right)$, and $1.1 \times 10^{10} \mathrm{~V} / \mathrm{cm}$ for ionizing the ninth electron to obtain $\mathrm{Kr}^{9+}$ ions. These electrical fields imply in the first case a laser intensity of $1.5 \times 10^{16} \mathrm{~W} / \mathrm{cm}^{2}$, and $1.6 \times 10^{17} \mathrm{~W} / \mathrm{cm}^{2}$ in the second case.

The more sophisticated model is a species-dependent tunneling ionization theory developed by Ammosov, Delone, and Krainov (ADK) [11]. The ADK model is based on a quasi-classical ac-tunneling theory and predicts the ionization rate of complex atoms or ions. It may be shown that the ionization rate depends very weakly on the laser frequency. Since the distortion of the atomic potential by the laser field yields a finite potential barrier, it is possible for the electron to quantum mechanically tunnel through this barrier and be ionized. This process is strongly nonlinear and shows a threshold-like behavior with laser intensity. The static field ionization rate is given by $[11,12]$

$$
\begin{aligned}
W=1.61 \omega_{a, u} \frac{Z^{2}}{n^{4.5}} & \left(10.87 \frac{Z^{3}}{n^{4}} \frac{E}{E_{L}}\right)^{2 n-1.5} \\
& \times \exp \left(-\frac{2}{3} \frac{Z^{3}}{n^{3}} \frac{E}{E_{L}}\right)
\end{aligned}
$$

where $\omega_{a . u}$ is the laser frequency in the atomic units, $E_{L}$ is the instantaneous strength of the laser electric field, $E$ is the atomic field strength, and $n=$ $Z(13.6 / U)^{\frac{1}{2}}$. The evolution of ion densities $n_{j}(t)$ for a particular charge state $j$ is then given by a series of first-order differential equations,

$$
\begin{gathered}
\frac{d n_{0}(t)}{d t}=-W_{1}(t) n_{0}(t) \\
\frac{d n_{j}(t)}{d t}=W_{j}(t) n_{j-1}(t)-W_{j+1}(t) n_{j}(t), \\
\quad \text { for } j=1,2, \ldots, n_{\max -1} \\
\frac{d n_{\max }(t)}{d t}=W_{\max }(t) n_{\max -1}(t)
\end{gathered}
$$

where $W_{j}(t)$ is the ionization rate for the production of charge state $j$. In this approximation, a stepwise tunneling ionization mechanism is considered as a dominant process. Note that the above described ionization model is commonly used $[12,13]$ because of good agreement with experimental results [10].

Fig. 1 shows the calculated temporal evolution of the relative population of charge states in argon for a $30-\mathrm{fs}$ laser pulse focused to an intensity of $5 \times 10^{16} \mathrm{~W} / \mathrm{cm}^{2}$. The ionization rate was set by the ADK model and the time evolution of charge states was determined by Eqs. (3)-(5). It can be seen that ions up to $\mathrm{Ar}^{8+}(\mathrm{Ne}-$

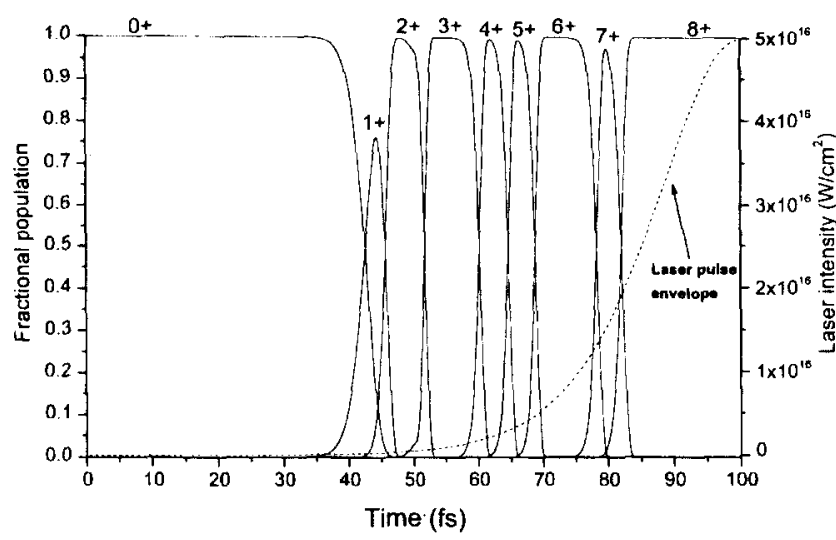

FIG. 1. Calculated temporal evolution of the relative population of argon charge states during interaction with $30-\mathrm{fs}$ laser pulse focused to peak intensity of $5 \times 10^{16} \mathrm{~W} / \mathrm{cm}^{2}$. The laser pulse peaks at $100 \mathrm{fs}$.

like) can be generated under this condition. Similar calculations were performed for krypton and xenon indicating production of ionic stages up to $\mathrm{Kr}^{8+}$ (Ni-like) and $\mathrm{Xe}^{8+}$ (Pd-like) for intensity above $1 \times 10^{16} \mathrm{~W} / \mathrm{cm}^{2}$. Since such a laser intensity is accessible using our experimental facility, the OFI theory can be further investigated experimentally.

\section{EXPERIMEN'T}

The experiment was conducted at KAIST using a femtosecond terawatt Ti:Sapphire laser system. The design of this terawatt laser has been described in detail elsewhere [14]. The laser is based on the chirped pulse amplification technique. The 17-fs pulses (energy $0.1 \mathrm{~nJ}$ ) from a mode-locked Ti:Sapphire oscillator $(\lambda=820 \mathrm{~nm})$ are stretched to a pulse length of approximately 200 ps before being injected into a 8pass preamlifier pumped by the second harmonic of a Nd:YAG laser. The output pulses containing energy of $3 \mathrm{~mJ}$ are further amplified by a 5-pass power amplifier to a maximum energy of $130 \mathrm{~mJ}$. The amplified radiation is then recompressed by a pair of diffraction gratings, thus delivering up to $60 \mathrm{~mJ}$ pulses of $30-\mathrm{fs}$ duration (FWHM) on the target. The laser can be operated at a repetition rate of up to $10 \mathrm{~Hz}$.

A simplified scheme of the experimental setup is shown in Fig. 2. Linearly polarized laser pulses were focused with a spherical mirror of $60 \mathrm{~cm}$ focal length into a gas jet of argon, krypton, and xenon from a pulsed nozzle (General Valve). The laser focus position was placed $250 \mu \mathrm{m}$ above the nozzle tip. The measured focal spot diameter was about $80 \mu \mathrm{m}$, so we could obtain peak intensities of up to mid- $10^{16} \mathrm{~W} / \mathrm{cm}^{2}$. The spectra were taken transversely (Fig. 2(a)) or longitudinally (Fig. 2(b)) to the direction of the pump laser 


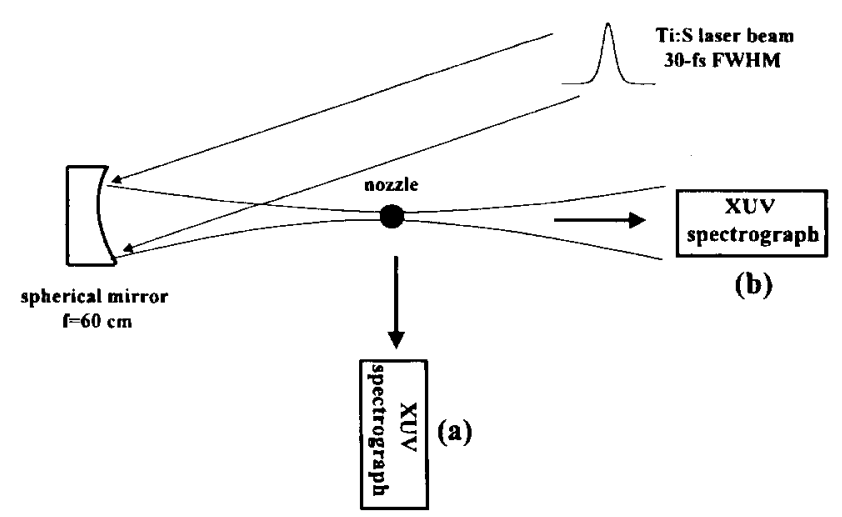

FIG. 2. Schematic of the experimental setup (top view) used for recording soft X-ray spectra from OFI plasmas in (a) transverse or (b) longitudinal geometry.

beam. The gas target was formed by pulsed injection of a small amount of gas from a high pressure valve through a circular nozzle (diameter of $200 \mu \mathrm{m}$ ) into a vacuum chamber. The valve was charged with gas at a backing pressure up to 15 bar. The gas density profiles were measured by a focusing method [15]. By varying backing pressure we were able to change the actual gas density in the interaction region. For example, backing pressure of 5 bar argon results in a peak density of about $2 \times 10^{18} \mathrm{~cm}^{-3}$ at a position $250 \mu \mathrm{m}$ above the nozzle tip.

Time integrated soft X-ray spectra were recorded by a space-resolving flat-field extreme ultraviolet (XUV) spectrograph [16]. It was coupled to a thinned, backside-illuminated charge-coupled device (CCD) camera. The covered wavelength range was from $40 \AA$ to $360 \AA$ and the spectral resolution was approximately $0.2 \AA$. However, in the case of longitudinal focusing geometry, an x-ray filter was used in front of CCD. A $0.1 \mu \mathrm{m}$ Al filter transmitted XUV radiation while rejecting visible stray light from the pump laser. Therefore a detectable spectral range in this case became from $180 \AA$ to $360 \AA$.

A typical longitudinal spectrum of krypton is shown in Fig. 3(a). The spectrum is dominated by Cu-like lines $\left(\mathrm{Kr}^{7+}\right)$, and five prominent lines from Ni-like ions $\left(\mathrm{Kr}^{8+}\right)$ were observed as well [17]. However, four of the $\mathrm{Ni}$-like lines have nearly the same wavelength as lines belonging to $\mathrm{Kr}^{7+}, \mathrm{Kr}^{6+}$, and $\mathrm{Kr}^{5+}$. It is interesting to note that the line at $326 \AA$ (distinct but rather weak) corresponds to the transition on which lasing was predicted $\left(3 d^{9} 4 d-3 d^{9} 4 p\right.$ in $\mathrm{Kr}^{8+}$ ions) [18]. Several weak lines from low ionization stages are also present. When the backing pressure dropped below 2 bar, bright highorder harmonics of the Ti:Sapphire pump laser were observed as shown in Fig. 3(b). Detailed interpretation of high harmonic emission can be found in [19].

In the longitudinal spectrum of xenon (Fig. 4), lines from Cd-like $\left(\mathrm{Xe}^{6+}\right)$ and $\mathrm{Ag}$-like $\left(\mathrm{Xe}^{7+}\right)$ ions were iden-
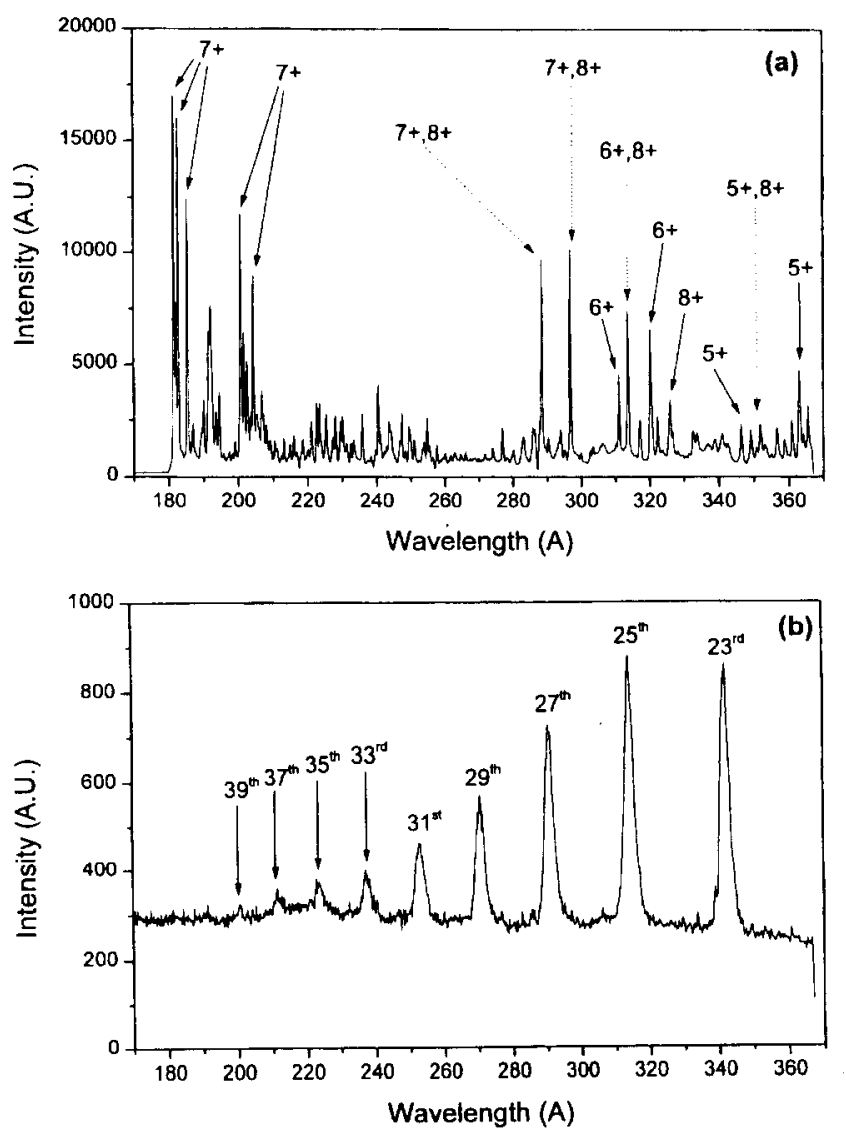

FIG. 3. Longitudinal spectra of krypton showing (a) strong emission on lines from $\mathrm{Kr}^{7+}$ and $\mathrm{Kr}^{8+}$ ions (gas density $\sim 2 \times 10^{18} \mathrm{~cm}^{-3}, 37 \mathrm{~mJ}, 50$ shots), and (b) odd harmonics of the laser frequency (gas density $\sim 2 \times 10^{17} \mathrm{~cm}^{-3}$, $37 \mathrm{~mJ}, 100$ shots).

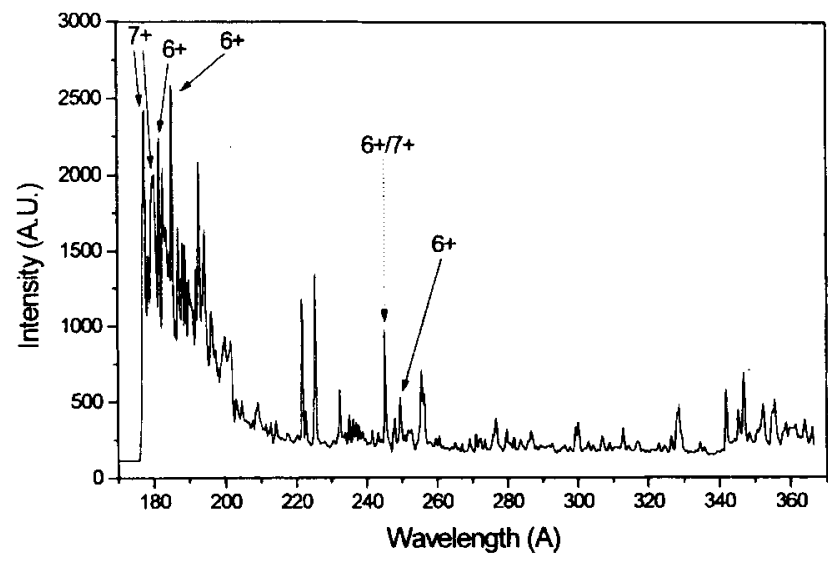

FIG. 4. Longitudinal spectrum of xenon showing Cd-like and Ag-like lines (gas density $\sim 2 \times 10^{18} \mathrm{~cm}^{-3}, 39 \mathrm{~mJ}, 200$ shots).

tified in the covered spectral region. The other observed lines remain unclassified due to the lack of reference spectral data. 

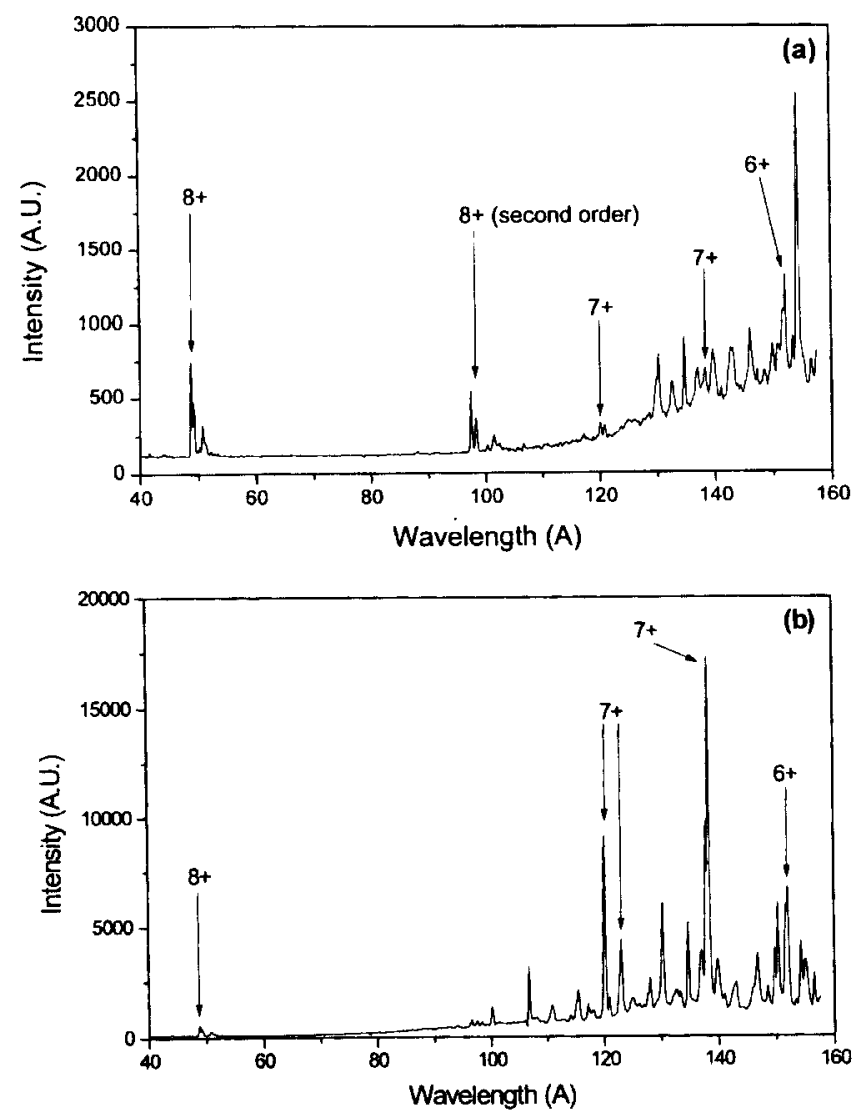

FIG. 5. Transverse spectra of argon showing (a) Ne-like (gas density $\sim 2 \times 10^{18} \mathrm{~cm}^{-3}, 49 \mathrm{~mJ}, 300$ shots), and (b) Na-like lines (gas density $\sim 4 \times 10^{18} \mathrm{~cm}^{-3}, 49 \mathrm{~mJ}, 20$ shots).

Fig. 5(a) shows a transverse spectrum of argon in the region of Ne-like $\left(\mathrm{Ar}^{8+}\right)$ resonance lines. The Ne-like $2 \mathrm{p}-3 \mathrm{~s}$ lines at $48.7 \AA$ and $49.2 \AA$ are prominent in the spectrum. After the backing pressure was increased to $10 \mathrm{bar}$, a different spectrum was observed(Fig. 5(b)). In this case, the density of gas in the interaction region reached a value of $\sim 4 \times 10^{18} \mathrm{~cm}^{-3}$. The Na-like $\left(\mathrm{Ar}^{7+}\right)$ lines show the highest intensity and $\mathrm{Mg}$-like $\left(\mathrm{Ar}^{6+}\right)$ line is relatively strong. The Nelike resonance lines are still present in the spectrum, however, much weaker compared to the lines from low ionization stages. We suppose that this effect may be caused by a significantly increased rate of three-body recombination to lower ionization states in higher density plasma.

\section{CONCLUSION}

We have presented a spectroscopic investigation of optical-field ionized noble gases. By focusing of 30-fs laser pulses of peak intensity up to $5 \times 10^{16} \mathrm{~W} / \mathrm{cm}^{2}$ into a gas jet of krypton, xenon, and argon, strong soft X-ray emission on lines of $\mathrm{Cu}$-like krypton, $\mathrm{Ni}$ - like krypton, Ag-like xenon, Na-like argon, and Ne-like argon was observed. This result is in good agreement with BSI and ADK theories.

\section{ACKNOWLEDGMENTS}

This research was supported by the Ministry of Science and Technology of Korea and in part by the Korea Science and Engineering Foundation.

\section{REFERENCES}

[1] P.B. Corkum, N.H. Burnett, and F. Brunel, Phys. Rev. Lett. 62, 1259 (1989).

[2] E. Fill, S. Borgström, J. Larsson, T. Starczewski, C.G. Wahlström, and S. Svanberg, Phys. Rev. E 51, 6016 (1995).

[3] G. Pretzler and Ernst E. Fill, Opt. Lett. 22, 733 (1997).

[4] G. Pretzler and Ernst E. Fill, Phys. Rev. E 56, 2112 (1997).

[5] T. Ditmire, T. Donnelly, R.W. Falcone, and M.D. Perry, Phys. Rev. Lett. 75, 3122 (1995)

[6] N.H. Burnett and G.D. Enright, IEEE J. Quantum Electron. 26, 1797 (1990).

[7] Y. Nagata, K. Midorikawa, S. Kubodera, M. Obara, H. Tashiro, K. Toyoda, and Y. Kato, Phys. Rev. A 51, 1415 (1995)

[8] D.V. Korobkin, C.H. Nam, and S. Suckewer, Phys. Rev. Lett. 77, 5206 (1996).

[9] B.E. Lemoff, G.Y. Yin, C.L. Gordon III, C.P.J. Barty, and S.E. Harris, Phys. Rev. Lett. 74, 1574 (1995).

[10] S. Augst, D.D. Meyerhofer, D. Strickland, and S.L. Chin, J. Opt. Soc. Am. B 8, 858 (1991)

[11] M.V. Ammosov, N.B. Delone, and V.P. Krainov, Sov. Phys.- JETP 64, 1191 (1986).

[12] B.M. Penetrante and J.N. Bardsley, Phys. Rev. A 43, 3100 (1991)

[13] P. Pulsifer, J.P. Apruzese, J. Davis, and P. Kepple, Phys. Rev. A 49, 3958 (1994)

[14] Y.H. Cha, Y.I. Kang, and C.H. Nam, CLEO/Pacific Rim '97 Tech. Dig. (1997), p.16.

[15] D.G. Lee and C.H. Nam, in preparation.

[16] I.W. Choi, J.U. Lee, and C.H. Nam, Appl. Opt. 36, 1457 (1997).

[17] B.E. Lemoff, Ph.D. "thesis, Department of Physics, Stanford University, 1994.

[18] B.E. Lemoff, C.P.J. Barty, and S.E. Harris, Opt. Lett. 19, 569 (1994).

[19] C.H. Nam, H.J. Shin, D.G. Lee, T. Mocek, Y.H. Cha, and K.H. Hong, "Significant blueshift of high harmonics generated in a femtosecond laser field," in Sixteenth International Conference on Atomic Physics, p438 (Windsor, Canada, August 1998).

[20] R.L. Kelly, J. Phys. Chem. Ref. Data 16, Suppl.1 (1987).

[21] J. Blackburn, P.K. Carroll, J. Costello, and G. O'Sullivan, J. Opt. Soc. Am. 73, 1325 (1983).

[22] T. Shirai, K. Okazaki, and J. Sugar, J. Phys. Chem. Ref. Data 24, (1995). 